Check for updates

Cite this: RSC Adv., 2019, 9, 9354

Received 28th December 2018 Accepted 18th March 2019

DOI: $10.1039 / c 8 r a 10605 c$

rsc.li/rsc-advances

\section{The medical application of terahertz technology in non-invasive detection of cells and tissues: opportunities and challenges}

\author{
Liu Yu, $\uparrow^{a}$ Liu Hao, $\uparrow^{\mathrm{ab}}$ Tang Meiqiong, ${ }^{\mathrm{a}}$ Huang Jiaoqi, ${ }^{\mathrm{a}}$ Liu Wei, ${ }^{\mathrm{a}}$ Dong Jinying, ${ }^{\mathrm{a}}$ \\ Chen Xueping, ${ }^{a}$ Fu Weiling ${ }^{+} \dagger^{\star a}$ and Zhang Yang $\dagger^{* a c}$
}

Terahertz $\left(\mathrm{THz}=10^{12} \mathrm{~Hz}\right)$ spectroscopy has shown great potential in biomedical research due to its unique features, such as the non-invasive and label-free identification of living cells and medical imaging. In this review, we summarized the advantages and progresses achieved in $\mathrm{THz}$ spectroscopy technology for blood cell detection, cancer cell characterization, bacterial identification and biological tissue discrimination, further introducing $\mathrm{THz}$ imaging systems and its progress in tissue imaging. We also highlighted the biological effects of $\mathrm{THz}$ radiation during its biological applications and the existing challenges and strategies to accelerate future clinical applications. The future prospects for $\mathrm{THz}$ spectroscopy will focus on developing rapid, label-free, and convenient biosensors for point-of-care tests and $\mathrm{THz}$ in vivo imaging.

\section{Introduction}

THz radiation typically is defined as $0.1-10 \mathrm{THz}$ of the electromagnetic spectrum $\left(3.33-333 \mathrm{~cm}^{-1}\right.$ in wavenumbers, or $30-$ $3000 \mu \mathrm{m}$ in wavelength), ${ }^{2}$ which lies between the microwave and infrared regions of the electromagnetic spectrum. In the past decade, numerous $\mathrm{THz}$ sources, detectors, and transmission or reflection technologies have been developed to explore the " $\mathrm{THz}$ gap" between photonics and electronics, ${ }^{5}$ and have been widely utilized in applications such as chemistry, materials science, biomedicine, security screening ${ }^{6}$ and communication. ${ }^{7}$

$\mathrm{THz}$ radiation has unique properties suited to biomedical research, especially which can excite low-frequency molecular vibrations, including hydrogen bonds, van der Waals or other non-bonded interaction. When $\mathrm{THz}$ radiation propagates through biomolecule, each biomolecule produces its characteristic spectral vibrational signature in the $\mathrm{THz}$ range. " $\mathrm{THz}$ fingerprint" could be utilized for identifying and characterizing objects based on $\mathrm{THz}$ spectroscopic measurements. ${ }^{8}$ Meanwhile, THz imaging has shown the crucial advantages in tissue detection in situ to distinguish normal and pathological tissues.

This review was divided into seven sections. The first section introduced basic concepts of $\mathrm{THz}$ radiation and its

\footnotetext{
${ }^{a}$ Department of Laboratory Medicine, Southwest Hospital, Third Military Medical University (Army Medical University), Chongqing 400038, China. E-mail: millen001@163.com; fwl@tmmu.edu.cn

${ }^{b}$ Department of Laboratory Medicine, The Second Hospital Affiliated to Dalian Medical University, Dalian 116023, China

${ }^{c}$ Department of Laboratory Medicine, Chongqing General Hospital, China

$\dagger$ The authors contributed equally.
}

fundamental mechanisms in biomedical application. The second section described the common types of $\mathrm{THz}$ spectroscopic instruments generally used in biological research. The third section surveyed the common THz imaging systems. The fourth section summarized the advantages and progress achieved in $\mathrm{THz}$ spectroscopic instruments for cell detection, including cancer cells, blood cells and bacterial cells. The fifth section summarized the application of THz spectroscopy and imaging in tissue detection. The sixth section provided an overview of the application of $\mathrm{THz}$ technology in bio-security. The last section addressed challenges and strategies to accelerate practical application on cell and tissue.

\section{THz spectroscopic instrument}

The THz spectroscopic instrument and imaging system are the essential conditions for detecting, analysis and imaging. The $\mathrm{THz}$ spectroscopy instrument can obtain the amplitude and phase information of the samples, which can be converted to optical parameters, such as refractive index, the extinction coefficient and so on, conveniently for studying the optical properties. ${ }^{9}$ Now, there are three main types of $\mathrm{THz}$ spectroscopy generally used in biological research, including Fourier transform spectroscopy (FTS), photomixing spectrometer and THz time-domain spectroscopy (THz-TDS).

\subsection{Fourier transform spectroscopy}

$\mathrm{THz}$ resonance spectroscopy was limited in the past, until the exploration of new sources and detectors. Recently, the new FTS with higher spectral and spatial resolution has been utilized for 
bacterial cells characterization in the sub-THZ range (10$\left.25 \mathrm{~cm}^{-1}\right) .{ }^{10}$ As the most common technique used for studying molecular resonance, FTS possess the prominent advantage of extremely wide spectral coverage, generally from $100 \mathrm{GHz}$ to 5 $\mathrm{THz}$, although its relatively poor signal-to-noise (SNR). A detail introduction can be found in a previous review about FTS. ${ }^{11}$

\subsection{Photomixing spectrometer}

The photomixing spectrometer generally contains a GaAs photoconductor, on which interdigitated metal electrodes are patterned with two frequency-offset lasers to generate continuous-wave. A schematic representation of a typical photomixing spectrometer is shown in Fig. 1A. ${ }^{12}$ This system contains two photomixers as transmitter and receiver, respectively. The photomixing spectrometer has a resolution of 500 $\mathrm{MHz}$ depended on the frequency step, whose dynamic range is also high, usually $70-80 \mathrm{~dB}$ around $0.1 \mathrm{THz}$ and $40-50 \mathrm{~dB}$ at 1.0 THz. ${ }^{13}$ Therefore, distinct signatures can be observed directly in the transmission spectra without further calculating the relevant spectral parameters. Although this technique requires a long measurement time, it's considered to be high accurate, inexpensive, and uncomplicated because of its huge spectral density and excellent frequency resolution. ${ }^{\mathbf{1 4}}$

\subsection{THz time-domain spectroscopy system}

THz-TDS uses short pulses of broadband THz radiation, that are typically generated using ultrafast laser pulses. ${ }^{15}$ Generally, a THz-TDS system contains a femtosecond laser, with a near 100 $\mathrm{MHz}$ repletion rate to further produce a series of $100 \mathrm{fs}$ laser pulses. Under an integration time of $1 \mathrm{~s}$, the THz-TDS system has an SNR between 50 and $60 \mathrm{~dB}$ in the bandwidth between 2 and $5 \mathrm{THz}$. With an acquisition time of $1 \mathrm{~min}$, the spectral resolution of a typical THz-TDS system is $50 \mathrm{GHz} .^{16,17}$

When the pulse train is split into a pump beam by a beam splitter, THz radiation and a probe beam to gate the detector are generated (Fig. 1B). ${ }^{16}$ The THz electric field can be obtained including the amplitude and phase information as a function of time. Thus, the THz-TDS system provides a time-resolved spectroscopic analysis and can effectively reject some common sources of noise.

\section{THz imaging system}

Recently, the $\mathrm{THz}$ imaging system has made rapid development, due to the crucial advantages of visualizations and high security, especially in medicine. Here, the two common $\mathrm{THz}$ imaging system will be introduced containing $\mathrm{THz}$ pulsed imaging (TPI) system and continuous-wave (CW) THz imaging system.

\subsection{THz pulsed imaging system}

The TPI system can achieve the imaging in the transmission and reflection mode. For example, a TPI reflection system (Fig. 2A) generally contains a femtosecond pulse laser, ${ }^{\mathbf{1 8}}$ operating with a biased photoconductive antenna to produce $\mathrm{THz}$ pulse, then that is collected, collimated, and focused onto the sample. Afterwards, the reflected and backscattered THz pulse is collected and focused onto an unbiased photoconductive antenna for the laser-gated $\mathrm{THz}$ detection. Different with TPI reflection system, the $\mathrm{THz}$ wave the penetrates into the sample in the TPI transmission system. ${ }^{19}$ In TPI reflection detection, the $\mathrm{THz}$ waveform is taken at many points over the sample surface, and is recorded as a function of optical time delay at each pixel.

Therefore, TPI system can provide three-dimensional information: the $x$ - and $y$-axis describe vertical and horizontal dimensions respectively and the $z$-axis represents the time-delay dimension. ${ }^{18}$ Despite TPI system requires a long time of scanning, it can record $\mathrm{THz}$ waveforms in the time domain, both including intensity and phase information, that can be used to acquire more details of the target. ${ }^{20}$

\subsection{Continuous-wave THz imaging system}

The CW THz imaging system also can achieve the imaging in the transmission and reflection mode. The Fig. 2B illustrates a $\mathrm{CW} \mathrm{THz}$ reflection imaging system that photomixes two $\mathrm{CW}$ lasers in a photoconductor as an example. ${ }^{2}$ The mixing of two above-bandgap (visible or near-infrared) wavelengths produces beating, that can modulate the conductance of a photoconductive switch at the $\mathrm{THz}$ difference frequency. Moreover, the farinfrared laser also can be used as the source. ${ }^{21}$ Different with $\mathrm{CW} \mathrm{THz}$ transmission imaging system, the $\mathrm{CW} \mathrm{THz}$ wave
A

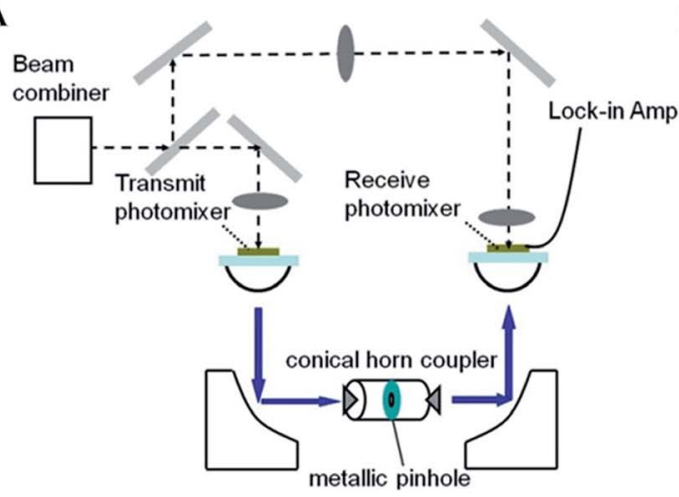

B

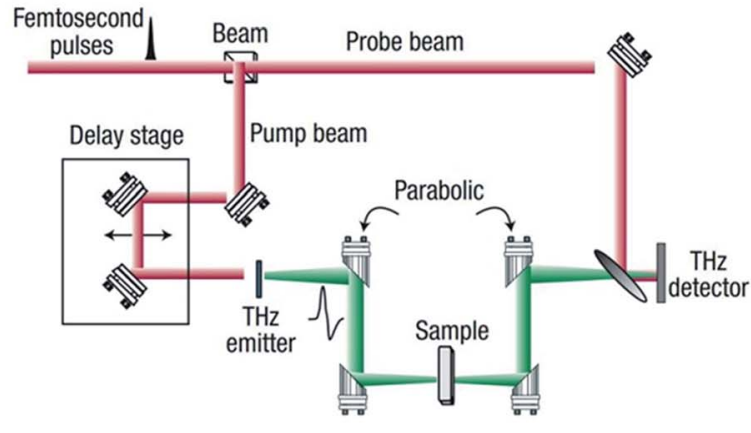

Fig. 1 Schematic illustration of THz spectroscopic instrument. (A) Photomixing spectrometer. (B) THz-TDS system. 
A

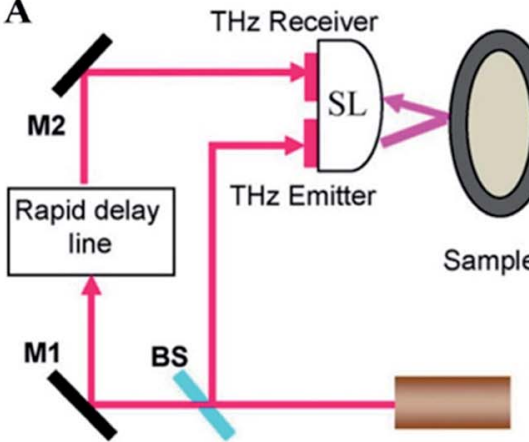

B

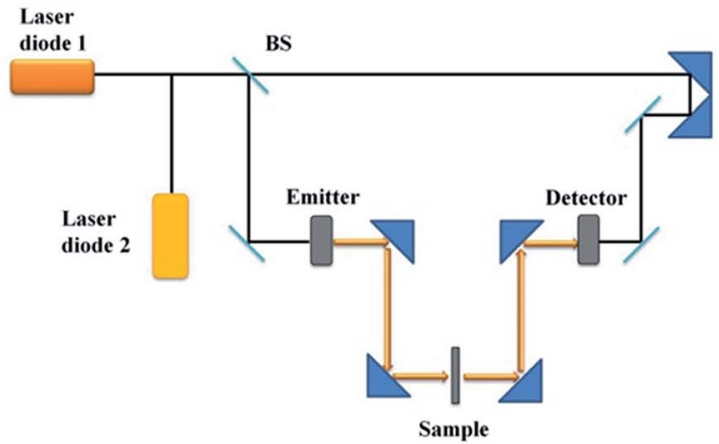

Fig. 2 Schematic illustration of THz imaging system. (A) TPI reflection system. (B) The CW THz imaging system in transmission geometry

produces reflections on the sample surface in the $\mathrm{CW} \mathrm{THz}$ reflection imaging system. ${ }^{22}$ For $\mathrm{CW}$ system, since the source spectrum is narrow and sometimes only the intensity information is useful, the data structures and post-processing are relatively simple, also making it confined to the applications. Meanwhile, it is possible to drive a whole CW system just by laser diodes, thus it can be made much compact and inexpensive.

\section{Applications of terahertz spectroscopy in cells detection}

Rapid and accurate detection is extremely important for early diagnosis, cell activity monitor, and human health care. The existing cell detection methods are mainly dependent on labeling technology, such as flow cytometry, fluorescence molecular tomography and multiphoton microscopy, with high sensitivity, precision and selectivity. However, inevitably, these methods require chemical or biological labeling, such as fluorophores or nuclides, which can directly affect the biological activity and function of living cells, fundamentally limiting the ability of existing cell detection to molecular level resolution. What's more, although the label-free cell detection method including various optical, electrochemical and piezoelectric technologies has been applied widely, potential radiation hazard and limited sensitivity still make them controversial. Overall, an alternative method for a label-free and non-invasive cell viability evolution is urgently needed for life sciences.

Based on emerging instruments, highly resolved $\mathrm{THz}$ spectroscopy is developing into an optical, non-invasive, label-free and reagent free technique, which can be utilized for detection and identification of living cells. Due to its unique features, $\mathrm{THz}$ spectroscopy has shown potential in living cell detection (Table 1):

\subsection{Terahertz spectroscopy of cancer cells}

Cancer is the most common cause of death since 2010 and one of the leading causes of mortality throughout the world. ${ }^{23}$ For cancer treatment, early diagnosis and regular monitoring are effective methods to reduce mortality. Recently, monitoring the slight change in cancer cells at early stage has been realized with $\mathrm{THz}$ pulsed spectroscopy based on identification of characteristic cancer spectral lines. ${ }^{24}$ The fact of apparent optical properties distinction between normal and diseased tissue cells has major significance in tumor identification on various stages. ${ }^{25}$ Moreover, the new THz spectroscopic system with high spectral and spatial resolution was demonstrated in application for measuring slight changes in cell monolayer, realizing quantitative analysis of cancer cells. ${ }^{25}$

Importantly, the application of THZ systems in hydrate state in human cancer cells has been reported widely. The hydration state in living cells has been proved to be associated with various cellular activities. Compared with normal cells, there are more free water and less bound water in the cancer cells. Importantly, the degree of cell hydration increases with the malignancy degree of cancer cells, indicating that intracellular hydration might be a primary factor in carcinogenesis. ${ }^{26}$ The phenomenon have been explained increased hydration could facilitate the acceleration of intracellular processes, for example respiration, that can enhance the competitive edge of a cancer cell for using nutrients. ${ }^{27}$

In the past decade, growing evidence has demonstrated the advantages of $\mathrm{THz}$ spectroscopy for detecting intracellular hydration in cancer cells. ${ }^{28}$ First and foremost, the relaxation process and intermolecular stretching vibration modes of intracellular water molecules are in the picosecond or subpicosecond range, which match exactly the range of the $\mathrm{THz}$ wave. Thus it have been demonstrated that the dielectric response of the cell can reflect particular water dynamics by $\mathrm{THz}$ spectroscopy (Fig. 3A). ${ }^{29}$ Secondly, THz spectroscopy can be used to evaluate the hydration status of tumor cells without complicated hydrogenation/deuterium replacement or cryogenic treatment. Thirdly, the electron energy of THz wave is low, so it will not cause obvious damage in the process of measuring the tumor cells and can observe the hydration state of the cells under physiological condition. Fourthly, $\mathrm{THz}$ time-domain attenuated total reflection (THz TD-ATR) spectroscopy can overcome the strong absorption of $\mathrm{THz}$ wave in liquid-phase environment, suitable for the detection of free water content in tumor cells.

Therefore, THz spectroscopy is highly suited for identifying cancer cells by signal characteristics, real-time monitoring the tiny structural changes and understanding the activities of 
Table 1 Features of $\mathrm{THz}$ spectroscopy for living cell detection

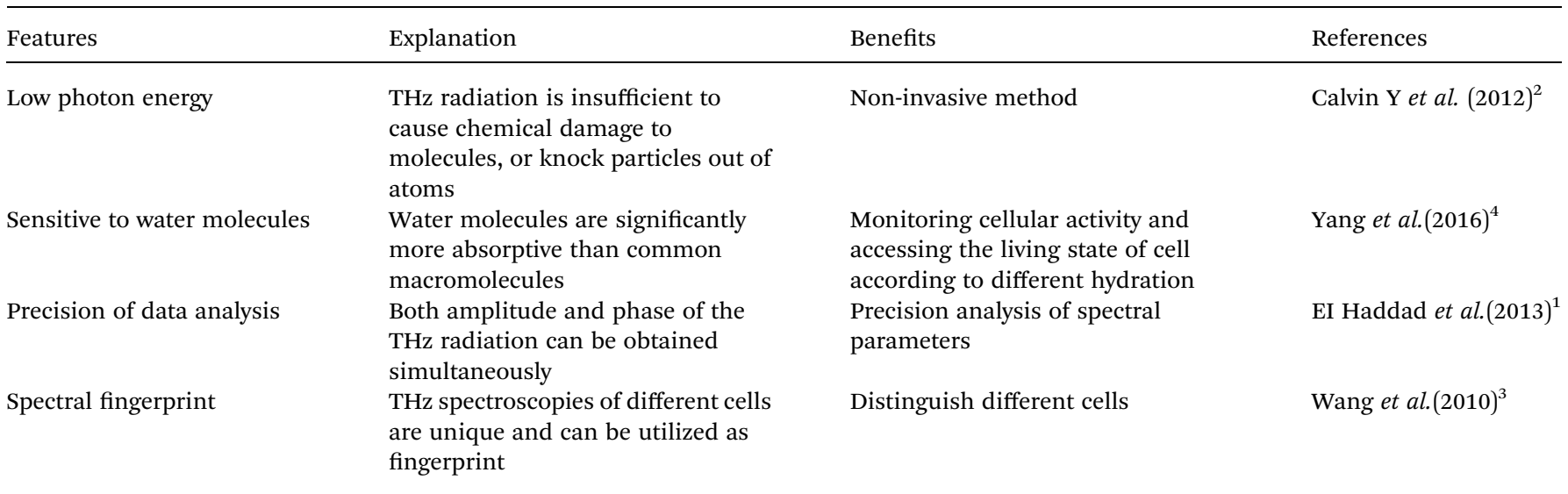

tumor cells by studying intracellular water dynamics in tumor cells. The entire process has the characteristics of real-time, rapid, label-free and non-invasive, which could lead to the development of novel tool for clinical analysis.

\subsection{Terahertz spectroscopy of blood cells}

Blood constants of qualitative and quantitative information about essential substances and waste products in the body can facilitate early diagnosis of several diseases, including cancer.
However, the study of $\mathrm{THz}$ spectroscopy to character blood cells has not been found widely in the literature. As $\mathrm{THz}$ radiation is highly absorbed by water which is one of the main constitutes of blood, the THz spectrum of blood are decided by water and not have any specific spectral feature useful for clinical application. Indeed, no significant difference in spectral between blood serum and water has been found between $50-650 \mathrm{~cm}^{-1}$.

Nevertheless, the researcher further investigated the spectroscopy features of various blood cells, attempting to find
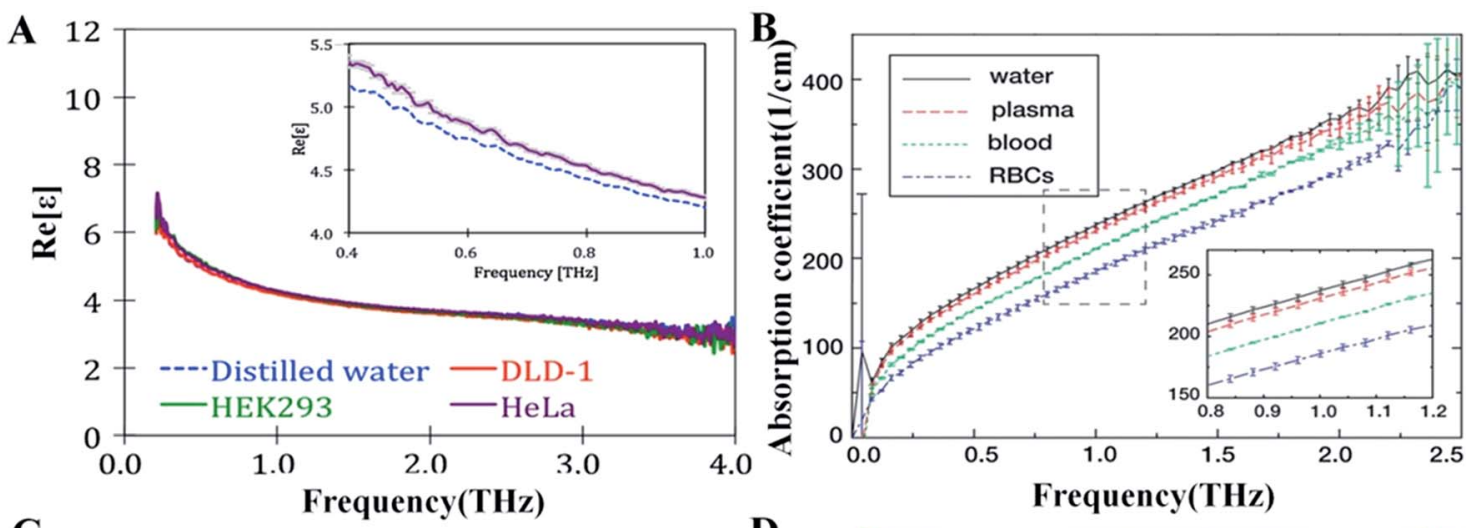

C

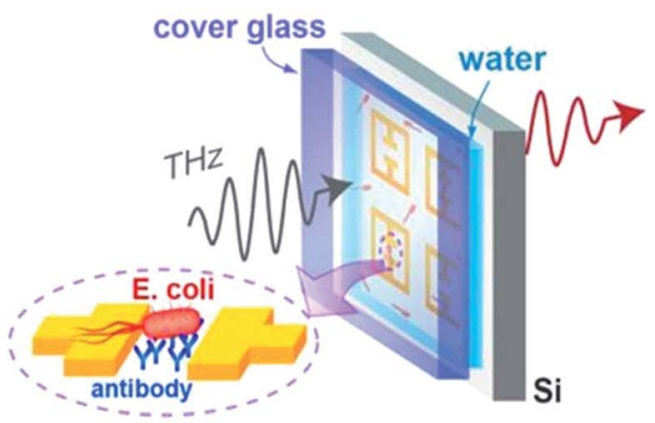

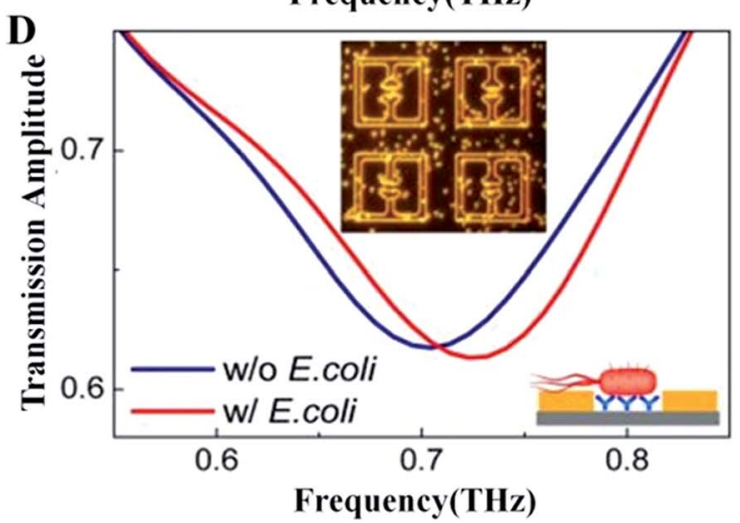

Fig. 3 (A) The real part of the complex dielectric constants of distilled water, DLD-1, HEK293 and HeLa detected by THz TD-ATR spectroscopy. (B) The absorption coefficient for water, plasma, blood and RBCs. (C) A schematic of specifically selective bacteria detection using metamaterial in solution environment. (D) THz transmission with and without the deposition of $E$. coli using the functionalized metamaterials in aqueous environments. 
certain signatures for particular types of biological cells. The study showed that different blood cells have their spectroscopy features in the $\mathrm{THz}$ range, and excellent linearity can be obtained between $\mathrm{THz}$ signals and erthrocyte concentrations (Fig. 3B), ${ }^{30}$ demonstrating a new method to qualitative and quantitative analysis of blood cells. Meanwhile, with the increasing interest and prospect on $\mathrm{THz}$ radiation for clinical application, we need to ensure that the energy absorption in human cells does not cause damage to cells.

\subsection{Terahertz spectroscopy of bacteria}

With the growing applications of $\mathrm{THz}$ technology for bacterial detection, researches on understanding of contributions from major structural components into the signature of the whole bacteria or spore have been raised. Based on small size and low absorption coefficient of the bacteria or spore, $\mathrm{THz}$ radiation can propagate through an entire object, allowing bacterial components contribute to the $\mathrm{THz}$ signature of objects. ${ }^{31}$ Subsequently, extensive research at the bacteria, spore and bacterial components aspects were conducted to realize qualitative and quantitative analyses of bacteria.

The spectral features of bacteria or spores mainly are determined by bacterial components, such as DNA, dipicolinic acid (DPA) and metabolites. In particular, the DNA of bacterial cells and spores might be essential for their $\mathrm{THz}$ signature. A study found that B. subtilis DNA and spores revealed common features in the THz spectra. ${ }^{32}$ Moreover, based on the apparent similarity for $E$. coli DNA and cells in the absorption spectra, it has clearly demonstrated the significant contribution of DNA to the overall spectra of the cell, and further was attributed to the strong absorption of hydrogen bonds in DNA.

The spectral features of DPA have also been studied in detail. As a unique component of bacillus spores, an increasing number of evidence indicated the possibility to detect Bacillus spores through DPA detection. DPA shares common spectral features between Bacillus thuringiensis and Bacillus globigii. ${ }^{33}$ Specifically, the distinct spectral signature at $1.54 \mathrm{THz}$ of DPA powder were in accord with the characteristic peak of B. subtilis spores in the $1.538 \mathrm{THz}^{34}$ Similarly, study on intracellular metabolites also revealed that $B$. subtilis cells and the intracellular metabolites have distinctive absorption features in the $\mathrm{THz}$ range, ${ }^{3}$ which may attribute to the strong absorption of riboflavin in the intracellular metabolites. ${ }^{35}$ However, although the absorption spectra of $B$. subtilis spores and cells were closely correlated, there were distinguishing features because spores have more compact structures than vegetative cells. ${ }^{36}$

The spectral features of bacteria have also been studied generally. The initial study using Bruker FTIR spectrometer demonstrated that transmission spectra can be measured at a temperature of $1.7 \mathrm{~K}$ between $3 \mathrm{~cm}^{-1}$ and $10 \mathrm{~cm}^{-1}$ for Erwinia herbacea and Bacillus subtilis lyophilized cells. Moreover, FTS in the frequency region of $10-25 \mathrm{~cm}^{-1}$ is sensitive enough to reveal characteristic spectral features of bio-cells in different environment, different types of bacteria, and viable and dead bacteria. ${ }^{37}$ In 2013, a high resolution of $0.3 \mathrm{GHz}$ Asia-Pacific Hertz vibration spectrum sensor prototype was built, making the required sample content from the order of milligram to nanogram level. ${ }^{31}$

In addition, qualitative and quantitative analyses of bacteria have been realized with the detection limit of $10^{4} \mathrm{cfu} \mathrm{ml}^{-1} \mathrm{ob}-$ tained through THz fiber by THz biosensors, which reached the sensitivity of the existing detection method. Quantitative detection of different bacteria has also been realized by $\mathrm{THz}$ plasmonic antennas, on the basis of their different dielectric responses in the $\mathrm{THz}$ range. ${ }^{38}$ Moreover, the sensitive detection of bacteria in both ambient and aqueous environments is feasible using $\mathrm{THz}$ metamaterials (Fig. 3C and D). ${ }^{39,40} \mathrm{~A} \mathrm{THz}$ metamaterial based on the SRR pattern of $550 \mu \mathrm{m}$ highresistance silicon substrate was used for high-sensitivity detection of bacteria in solid and liquid phase. ${ }^{39,40}$ Another application of $\mathrm{THz}$ biosensing is to provide an insight into the spectral differences between living and dead bacteria as a result of the irreversible transformation of cellular component structures. ${ }^{36}$

\subsection{Prospective opinion}

Despite the enormous potential for clinical detection on the cell and bacteria, $\mathrm{THz}$ spectroscopy is still at early stage of development. The "Spectral Fingerprint Database" is a prerequisite for the identification of unknown object. However, it's very essential to establish a standardized detection system that can break through the bottleneck. On the other hand, it is difficult for the researchers to solve the problem of screening the target spectral fingerprints from complex backgrounds and eliminate the interference signals. Although the existing Fourier transform infrared spectroscopy and THz-TDS systems failed to meet the requirements, the $\mathrm{THz}$ plasmonic antennas and metamaterial may realize, on the basis of their different dielectric responses in the $\mathrm{THz}$ range. Therefore, the establishment of high sensitivity and high specificity $\mathrm{THz}$ sensor will be the future development direction of $\mathrm{THz}$ wave for the cell detection.

\section{Applications of terahertz spectroscopy and imaging in tissue}

\subsection{Terahertz spectroscopy of tissue}

With the elevated interest to develop THz technology, it has facilitated that THz spectroscopy can be applied to detect and differentiate healthy, dehydrate, burned and pathological tissues. Due to the unprecedented ability to operate on the same timescale with the vibrational motion of water and biomolecules on the subpicosecond to picoscenond, THz spectroscopy permit time-resolved of these dynamic. ${ }^{\mathbf{4 1 , 4 2}}$ Moreover, $\mathrm{THz}$ spectroscopy also permits the measurement of amplitude and phase information, which is directly related to the absorption coefficient and index of refraction of tissues. THz spectroscopy shows its potential for tissue detection because of these features.

However, the major component of fresh tissue is water, such as skin tissues ( $-70 \%$ by volume), exhibiting strong absorption at $\mathrm{THz}$ frequencies $\left(u_{\mathrm{a}}=300 \mathrm{~cm}^{-1}\right.$, at $\left.1.5 \mathrm{THz}\right){ }^{42}$ To avoid the effects of strong optical absorption of water, different tissue 
preparation methods have been developed, such as paraffin, frozen and formalin fixed tissues. Recently, paraffin-embedded tissues from cancer patients appeared to display higher absorption coefficients and refractive index than healthy tissues (Fig. 4A). ${ }^{43}$ Furthermore, snap-frozen tissues were also frequently measured to reduce the influence of water, and numerous data revealed that Alzheimer's patients' tissues displayed less absorption than normal tissues. ${ }^{44}$

Similarly, the strong optical absorption of water cause only a few millimeters penetration in biological tissues, so the application of $\mathrm{THz}$ in vivo spectroscopy still remain in the surface of biological tissues. Meanwhile, the property of strong water absorption is also beneficial because it provides a sensitive method for soft-tissue contrast. Significantly different absorption coefficients and refractive indexes can be shown among various skin tissues (Fig. 4B). ${ }^{45}$ Specifically, the different dielectric parameters of healthy skin, dysplastic nevus and nondysplastic nevus clearly demonstrates the possibility for early, non-invasive diagnostics of pigmentary skin nevus. ${ }^{46}$

\subsection{Terahertz imaging of tissue}

THz transmission-based imaging has been used to examine the dynamics of water, biomolecules and biological tissues. Similarly to $\mathrm{THz}$ spectroscopy of tissue, the $\mathrm{THz}$ transmission-based imaging is generally performed on either lyophilized tissues or thin tissue slices $(100 \mu \mathrm{m}){ }^{47-49}$ The main problem of the method is greatly affected by the hydration level and morphology of the tissue, reducing the accuracy of the measurements. For exactly these factors, reflection geometries are preferred for both in vivo and in vitro $\mathrm{THz}$ measurements of biological tissues.

Several reflection-based $\mathrm{THz}$ imaging systems have been developed for the non-invasive detection of bio-tissues which are widely used to compare water content changes, to diagnose specific types of cancer and to assess the depth and severity of burned tissues. Because the cancerous or other diseased tissues can contain higher water content as a result of increased vascularity or edema, $\mathrm{THz}$ imaging has shown potential in differentiating tissue types. Meanwhile different healthy organs have been distinguished between rat tissues by TPI reflection system. Moreover, a recent simulation study found that InNenhanced $\mathrm{THz}$ imaging enables ultrahighly sensitive diagnosis of early-stage skin cancer based on tiny water content changes. ${ }^{50}$

The water contents of blood vessel and corneal tissue were also clearly resolved using a $\mathrm{THz}$ imaging system, promising in vivo applications for the long-term monitoring the blood change and quantitative measurements of corneal tissue water content. ${ }^{51-53}$ Furthermore, the difference among various tissues is still significant after formalin fixing, suggesting that the difference is not only due to changes in water content, but also structural changes. Moreover, based on higher $\mathrm{THz}$ reflection intensities, fresh human early gastric cancer tissues have been distinguished from normal mucosa, that fits well with the pathological images, except for signet ring cell carcinoma (Fig. 5A)..$^{54}$ Notably, it's successful that the imaging of human hepatocellular carcinoma tissue has been obtained using CW THz digital in-line holography in the absorption and phase-shift distributions (Fig. 5B).$^{55}$ What's more, by rejecting Fresnel reflections without the sample information coming from the glass-air interface, the cross-polarized $\mathrm{THz}$ image of nonmelanoma skin cancer displays the approximate location of the tumor clearly. ${ }^{56}$

Due to its limited penetration depth, THz in vivo imaging has mainly been applied to epidermal tissue and achieved several meaningful breakthroughs. Uniformly, water absorption plays a dominant role in shaping the variable angle ellipsometry or polarized reflectometry $\mathrm{THz}$ spectrum in a human skin layer model..$^{57,58}$ Experimentally, dynamic changes of reflected signal in rat skin burns were clearly observed by $\mathrm{THz}$ in vivo imaging, which satisfies an imperious need in skin burn assessment. ${ }^{50}$ What's more, it's potential to integrate the $\mathrm{THz}$ imaging into regular endoscopic system, which promotes the development of THz technology in clinical application. ${ }^{59}$ At present, the hypothesis have been performed
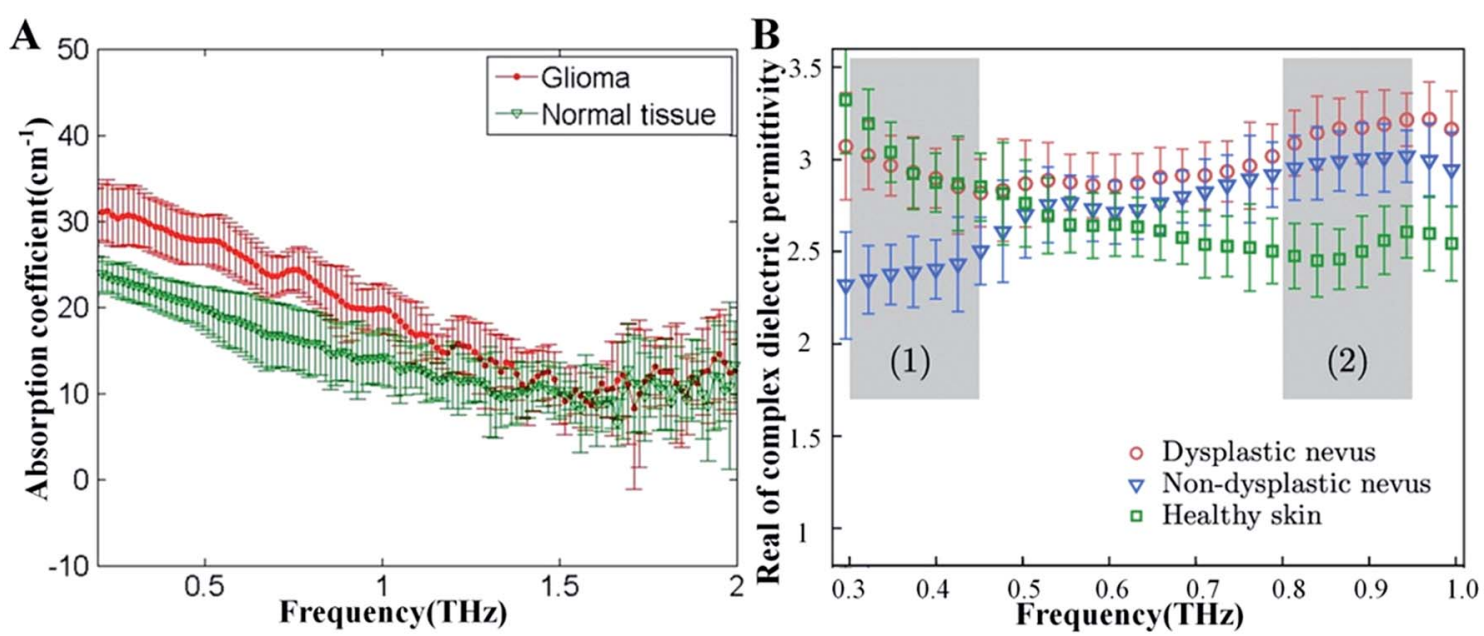

Fig. 4 The absorption coefficients of tissues. (A) The absorption coefficient of glioma and normal tissue. (B) The real of the THz dielectric permittivity of dysplastic nevus, non-dysplastic nevus and healthy skin. 


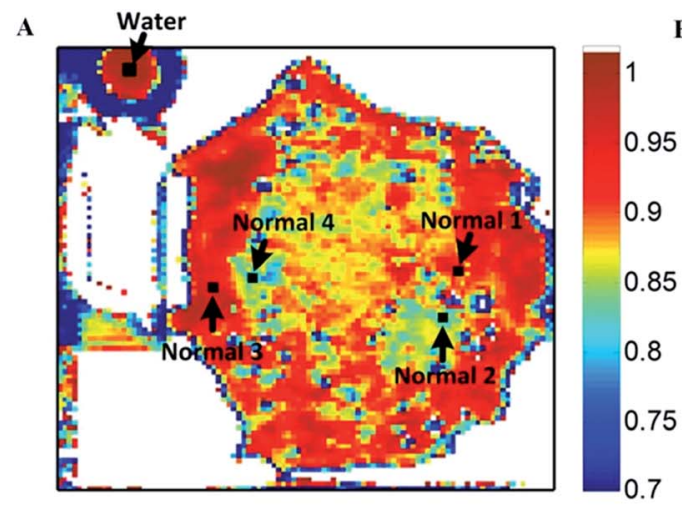

B

Fig. 5 The THz image of tissues. (A) Tissue from early gastric cancer. (B) THz in-line hologram of human hepatocellular carcinoma tissue by phase-shift distribution reconstructing.

by four procedure in sequence, showing the sensitive screening for colorectal cancer. ${ }^{60}$

\subsection{Prospective opinion}

For tissue detection, $\mathrm{THz}$ imaging has great potential for clinical application, whose critical advantage is without radiation hazard, compared with traditional imaging methods, such as X-ray scanning, magnetic resonance imaging (MRI) and so on. Moreover, for superficial neoplasms, the penetration depth of $\mathrm{THz}$ imaging is more matched than the MRI, with the ability to develop clinical imaging detection in realtime, non-destructive and visual that can combine with $\mathrm{THz}$ spectroscopy or endoscopic system. Despite these impressive breakthroughs, some inherent limitations in tissue detection by $\mathrm{THz}$ technology still exist, the most serious of which is imperfect formalized standard for handling and preserving samples. ${ }^{47}$

\section{The bio-security of THz technology applications}

With the rapid development of THz technology, investigating the biological effects of $\mathrm{THz}$ radiation is becoming more critical at the level of organs, tissues, cells and biomolecules. At the organism level, THz irradiation can cause several effects, but it poses a slight potential health hazard. A study in a mouse model found that high-power density $\mathrm{THz}$ exposure (0.15 THz, $3 \mathrm{~mW} \mathrm{~cm} \mathrm{~cm}^{-2}, 60 \mathrm{~min}$ ) can increase levels of depression. ${ }^{61}$ Similar studies in mouse skin also revealed that $\mathrm{THz}$ irradiation can cause inflammatory responses $(2.7 \mathrm{THz}$, $260 \mathrm{~mW} \mathrm{~cm}{ }^{-2}, 30 \mathrm{~min}$ ) and perturbation of the woundhealing $\left(2.5 \mathrm{THz}, 0.32 \mu \mathrm{W} \mathrm{cm}^{-2}, 60 \mathrm{~min}\right),{ }^{62,63}$ there was no change in temperature of the skin of mouse that was exposed to $\mathrm{THz}$ radiation. Moreover, no obvious temperature change and tissue damage were observed in Drosophila melanogaster and rats under THz irradiation. . $^{64,65}$

The influences of $\mathrm{THz}$ irradiation at tissues and cells have also been studied indicating that tissue damage and cell death can result from the thermal effects of high-power densities. An in vitro study found that high-power density THz exposure (0.1-1.0 THz, 2000-14 $000 \mathrm{~mW} \mathrm{~cm}^{-2}$ ) could produce tissue damage in wet chamois cloths at a damage threshold of $7160 \mathrm{~mW} \mathrm{~cm}{ }^{-2}$, 66 with an obvious temperature rise. However, low-power density $\mathrm{THz}$ exposure $(1.89 \mathrm{THz}$, $189.92 \mathrm{~mW} \mathrm{~cm}^{-2}$ ), can't induce tissue damage in porcine skin. Besides, similar studies on cells revealed that high-power density $\left(2.52 \mathrm{THz}, 84.8 \mathrm{~mW} \mathrm{~cm}{ }^{-2}\right) \mathrm{THz}$ exposure induced almost $10 \%$ cell death and increased the temperature by $3{ }^{\circ} \mathrm{C} .{ }^{67}$ By contrast, low-power density THz exposure (0.02-0.37 $\mathrm{mW} \mathrm{cm}^{-2}$ ) caused no adverse effects on the morphology, proliferation, or differentiation of human ocular cell lines and embryonic stem cells. ${ }^{68}$ It should be mentioned that cell membrane structural and functional properties can be altered under $\mathrm{THz}$ irradiation under 30 to $300 \mathrm{GHz}$ via non-thermal mechanism. ${ }^{69}$

Several researchers have proposed that low-level THz irradiation may induce non-thermal effects at the molecular level, wherein $\mathrm{THz}$ radiation induced low-frequency collective biomolecular vibrations..$^{70}$ At a molecular level, when biological tissues were exposed to $\mathrm{THz}$ irradiation, biological molecules underwent conformational changes and/or denature, such as DNA damages, ${ }^{71}$ gene expression alterations, ${ }^{72}$ and protein changes..$^{73}$ However, a recent study indicated that no obvious DNA damages or negligible gene changes in the 2.3 THz. ${ }^{74}$ However, the energy of the THz pulses used in typical $\mathrm{THz}$ biomedical applications and representative laser-based THz-TDS systems was several orders of magnitude lower than these reported above. Thus, during real applications, temperature increase in the human body would be negligible. ${ }^{70}$

To sum up, whether $\mathrm{THz}$ radiation can threat to biosecurity is still inconclusive. According to the present studies, the biological effects of $\mathrm{THz}$ radiation depend on irradiation parameters including frequency, power, and time and so on, and types of the biological target. Of particular concern is that $\mathrm{THz}$ radiation at high-power density can cause thermal effects, which is essential for fully understanding the biological effects. Although the common $\mathrm{THz}$ pulses used in typical $\mathrm{THz}$ laboratory is too low to cause 
serious effects, it's very necessary to properly protect security of experimenters.

\section{Challenges and future directions}

Although $\mathrm{THz}$ technology has shown great potential in biomedical research, there are still several challenges need to be solved before this technology can be widely applied in clinical. Firstly, the detection sensitivity of $\mathrm{THz}$ wave does not meet the special requirements of living cell detection, because the physical diffraction limit and $\mathrm{THz}$ wavelength $(0.03-3 \mathrm{~mm})$ is larger than the resolution of live cell detection in the micron or even nano-order. Therefore, how to break through the diffraction limit and improve the detection sensitivity always is the technical bottleneck faced with $\mathrm{THz}$ technology in the biological detection.

Secondly, the specificity of detection of THz waves is limited by the complexity of detecting environmental conditions. Biological samples often contain different living cells, and a large number of macromolecules in both inside and outside the cell, which always can produce some serious signal interference, leading the signal interference and annihilation of the target. Therefore, how to use effective technical means to analyze the spectral characteristics and images from the detection results is a scientific problem that $\mathrm{THz}$ technology must be solved.

Thirdly, the water sensitivity of the $\mathrm{THz}$ wave is a technical problem in living cell detection. As the $\mathrm{THz}$ wave is very sensitive to water, the previous studies are mostly limited to the analysis of molecules, cells and tissues under solid or dry conditions. The water-rich human biological samples and the water vapor interference in the environment are the major problems in detection. Thus, the cell's THz wave detection must overcome the water sensitivity of the detection signal interference and annihilation.

Last but not least, biological safety of $\mathrm{THz}$ irradiation is still a lack of scientific experimental basis. THz wave photon energy is very low, once considered to be safe on cells, tissues, and even the human body. However, recent studies have shown that $\mathrm{THz}$ irradiation with specific intensity and irradiation time can lead to a certain degree of variation in the genome, proteome and transcriptome of cells and tissues, so researchers began to reconsider the bio-safety of THz irradiation in biomedical applications areas.

In spite of these challenges, the corresponding breakthroughs have been made in recent years. Taking advantage of evanescent wave, the $\mathrm{THz}$ attenuated total reflection spectroscopy has achieved the non-invasive, in situ, and real-time investigation of cytoplasm leakage and the monitoring of cell hydration state. In addition, based on kinds of burgeoning meta-materials, the biosensors for qualitative and quantitative detection of bacterial and cell hydration state analysis are developing rapidly, expected to supplement the routine clinical detections. ${ }^{75}$ Recently, THz technology has been combined with conventional endoscopic system, prospectively achieving the imaging in real-time and visual during non-destructive detection and surgical operations. Thus, THz technology has made the unprecedented progress, and is being developed into clinical application step by step.

\section{Conflicts of interest}

There are no conflicts to declare.

\section{Acknowledgements}

This work was supported by the National Key Basic Research Program of China (2015CB755400); the National Natural Sciences Foundation of China (81430054) and the Logistics Scientific Research Project (BWS13C013, AWS17J010, 2016XYY08).

\section{References}

1 J. E. Haddad, B. Bousquet, L. Canioni and P. Mounaix, TrAC, Trends Anal. Chem., 2013, 44, 98-105.

2 C. Yu, S. Fan, Y. Sun and E. Pickwell-Macpherson, Quant. Imaging. Med. Surg., 2012, 2, 33-45.

3 C. Wang, J. Gong, Q. Xing, Y. Li, F. Liu, X. Zhao, L. Chai, C. Wang and A. M. Zheltikov, J. Biophotonics, 2010, 3, 641645.

4 X. Yang, D. Wei, S. Yan, Y. Liu, S. Yu, M. Zhang, Z. Yang, X. Zhu, Q. Huang and H. L. Cui, J. Biophotonics, 2016, 9, 1050-1058.

5 D. H. Auston and M. C. Nuss, IEEE J. Quantum Electron., 2002, 24, 184-197.

6 I. Duling and D. Zimdars, Nat. Photonics, 2009, 3, 630-632.

7 V. Petrov, J. Kokkoniemi, D. Moltchanov, J. Lehtomäki and

Y. Koucheryavy, Physical Communication, 2017, 22, 9-18.

8 T. Globus, I. Sizov, J. Ferrance, A. Jazaeri, J. Bryant, A. Moyer, B. Gelmont, M. Kester and A. Bykhovski, Convergent Sci. Phys. Oncol., 2016, 2, 045001.

9 S. Ping and Z. Yun, Opt. Quantum Electron., 2016, 48, 27.

10 T. Globus, A. Moyer, B. Gelmont, T. Khromova, I. Sizov and J. Ferrance, IEEE Sens. J., 2013, 13, 72-79.

11 R. J. Markovich and C. Pidgeon, Pharm. Res., 1991, 8, 663.

12 W. Zhang, E. R. Brown, M. Rahman and M. L. Norton, Appl. Phys. Lett., 2013, 102, 219-222.

13 W. Zhang, E. R. Brown, L. Viveros, K. P. Burris and S. C. Jr, J. Biophotonics, 2015, 7, 818-824.

14 S. Preu, G. H. Döhler, S. Malzer, L. J. Wang and A. C. Gossard, J. Appl. Phys., 2011, 109, 4-1548.

15 C. Fattinger and D. Grischkowsky, Appl. Phys. Lett., 1988, 53, 1480-1482.

16 B. Ferguson and X.-C. Zhang, Physics, 2003, 1, 26-33.

17 A. Redo-Sanchez, N. Laman, B. Schulkin and T. Tongue, Int. J. Infrared Millimeter Waves, 2013, 34, 500-518.

18 Y. C. Shen, Int. J. Pharm., 2011, 417, 48-60.

19 P. C. Ashworth, P. M. Emma, P. Elena, S. E. Pinder, A. D. Purushotham, P. Michael and V. P. Wallace, Opt. Express, 2009, 17, 12444-12454.

20 K. Mcclatchey, M. T. Reiten and R. A. Cheville, Appl. Phys. Lett., 2001, 79, 4485-4487.

21 P. Doradla, K. Alavi, C. Joseph and R. Giles, J. Biomed. Opt., 2014, 19, 080501.

22 C. S. Joseph, A. N. Yaroslavsky, T. M. Goyette and R. H. Giles, Proc. SPIE-Int. Soc. Opt. Eng., 2012, 8261, 26. 
23 W. Chen, R. Zheng, P. D. Baade, S. Zhang, H. Zeng, F. Bray, A. Jemal, X. Q. Yu and J. He, Ca-Cancer J. Clin., 2016, 66, 115132.

24 E. A. Strepitov, E. P. Liakhov, N. S. Balbekin, M. K. Khodzitsky, O. A. Smolyanskaya, A. S. Trulyov and M. K. Serebryakova, Medical Laser Applications and LaserTissue Interactions VII, 2015, vol. 9542, 95420.

25 H.-B. Liu, G. Plopper, S. Earley, Y. Chen, B. Ferguson and X.-C. Zhang, Biosens. Bioelectron., 2007, 22, 1075-1080.

26 C. Martin, Nat. Rev. Mol. Cell Biol., 2006, 7, 861-866.

27 E. O. Potma, W. P. D. Boeij and D. A. Wiersma, Biophys. J., 2001, 80, 3019-3024.

28 P. Ball, ChemPhysChem, 2010, 9, 2677-2685.

29 K. Shiraga, Y. Ogawa, T. Suzuki, N. Kondo, A. Irisawa and M. Imamura, Int. J. Infrared Millimeter Waves, 2014, 35, 493-502.

30 C. B. Reid, G. Reese, A. P. Gibson and V. P. Wallace, IEEE Journal of Biomedical and Health Informatics, 2013, 3, 363367.

31 T. Globus, A. M. Moyer, B. Gelmont, T. Khromova, M. I. Lvovska, I. Sizov, J. Ferrance, T. Globus, A. M. Moyer and B. Gelmont, IEEE Sens. J., 2013, 13, 72-79.

32 A. Bykhovski, X. Li, T. Globus, T. Khromova, B. Gelmont, D. Woolard, A. C. Samuels and J. O. Jensen, Proc. SPIE-Int. Soc. Opt. Eng., 2005, 5995.

33 M. J. Fitch, C. Dodson, D. S. Ziomek and R. Osiander, Proc. SPIE, 2004, 5584, 16-22.

34 B. Yu, A. Katz and R. R. Alfano, Proc. SPIE, 2005, 5727.

35 K. E. Tyo, H. S. Alper and G. N. Stephanopoulos, Trends Biotechnol., 2007, 25, 132-137.

36 T. Globus, D. L. Woolard, T. Khromova, R. Partasarathy, A. Majewski, R. Abreu, J. L. Hesler, S. K. Pan and G. Ediss, Proc. SPIE-Int. Soc. Opt. Eng., 2004, 5411, 25-32.

37 T. Globus, D. Woolard, T. W. Crowe, T. Khromova, B. Gelmont and J. Hesler, Optical Technologies for Industrial, Environmental, and Biological Sensing, 2004.

38 M. Anna, M. Andrey, N. Andy, C. Raja, S. Olga, Z. Mohammed and S. Maksim, Opt. Express, 2012, 20, 5344-5355.

39 A. Berrier, M. C. Schaafsma, G. Nonglaton, J. Bergquist and J. G. Rivas, Biomed. Opt. Express, 2012, 3, 2937-2949.

40 S. J. Park, J. T. Hong, S. J. Choi, H. S. Kim, W. K. Park, S. T. Han, J. Y. Park, S. Lee, D. S. Kim and Y. H. Ahn, Sci. Rep., 2014, 4, 4988.

41 D. Russo, G. Hura and T. Head-Gordon, Biophys. J., 2004, 86, 1852-1862.

42 K. Kodo, O. Yuichi, W. Yuuki and I. Hiroyuki, Opt. Express, 2003, 11, 2549-2554.

43 M. Kun, C. Tu-Nan, C. Tao, Z. Li-Guo, L. Qiao, L. Zhao, L. Fei, Z. Sen-Cheng, L. Ze-Ren and F. Hua, J. Biomed. Opt., 2014, 19, 077001.

44 G. M. Png, R. Flook, W. H. Ng and D. Abbott, Electron. Lett., 2009, 45, 343-345.

45 E. Ibtissam, J. A. Grundt, T. Melissa, B. L. Ibey, T. Thomas, L. Min, X. Hao and G. J. Wilmink, J. Biomed. Opt., 2013, 18, 120503.

46 K. I. Zaytsev, K. G. Kudrin, V. E. Karasik, I. V. Reshetov and S. O. Yurchenko, Appl. Phys. Lett., 2015, 106, 053702.
47 G. Png, J. Choi, B. W.-H. Ng, S. Mickan, D. Abbott and X. Zhang, Phys. Med. Biol., 2008, 53, 3501.

48 R. S. Singh, P. Tewari, J. L. Bourges, J. P. Hubschman, D. B. Bennett, Z. D. Taylor, H. Lee, E. R. Brown, W. S. Grundfest and M. O. Culjat, Conf. Proc. IEEE Eng. Med. Biol. Soc., 2010, 2010, 3021-3024.

49 J. Y. Suen, P. Tewari, Z. D. Taylor, W. S. Grundfest, H. Lee, E. R. Brown, M. O. Culjat and R. S. Singh, Stud. Health Tech. Informat., 2009, 142, 364-368.

50 M. Ney and I. Abdulhalim, J. Biomed. Opt., 2015, 20, 125007. 51 T. Tzu-Fang, Y. Szu-Chi, S. Yuan-Ta, T. Yuan-Fu, W. TzungDau and S. Chi-Kuang, Opt. Express, 2015, 23, 25058.

52 Z. D. Taylor, J. Garritano, S. Sung, N. Bajwa, D. B. Bennett, B. Nowroozi, P. Tewari, J. W. Sayre, J. P. Hubschman and S. X. Deng, IEEE Trans. Terahertz Sci. Technol., 2015, 5, 184-196.

53 B. David B, T. Zachary D, T. Pria, S. Rahul S, C. Martin O, G. Warren S, S. Daniel J, J. R Duncan, H. Jean-Pierre and B. Elliott R, J. Biomed. Opt., 2011, 16, 057003.

54 H. P. Chan, G. M. Lee, H. Kim, J. S. Suh, J. H. Son, S. K. Noh, K. L. Sang, S. H. Kim, S. J. Oh and S. Haam, Biomed. Opt. Express, 2015, 6, 1398.

55 L. Rong, T. Latychevskaia, C. Chen, D. Wang, Z. Yu, X. Zhou, Z. Li, H. Huang, Y. Wang and Z. Zhou, Sci. Rep., 2015, 5, 8445.

56 C. S. Joseph, R. Patel, V. A. Neel, R. H. Giles and A. N. Yaroslavsky, J. Biophotonics, 2014, 7, 295-303.

57 N. Michael and I. Abdulhalim, Opt. Lett., 2010, 35, 3180.

58 M. Ney and I. Abdulhalim, J. Biomed. Opt., 2011, 16, 067006.

59 P. Doradla, Terahertz Endoscopic System for Cancer Detection, 2015.

60 P. Doradla, C. Joseph and R. H. Giles, World Journal of Gastrointestinal Endoscopy, 2017, 9, 7-19.

61 V. F. Kirichuk, N. V. Efimova and E. V. Andronov, Bull. Exp. Biol. Med., 2009, 148, 746-749.

62 Y. Hwang, J. Ahn, J. Mun, S. Bae, Y. U. Jeong, N. A. Vinokurov and P. Kim, Opt. Express, 2014, 22, 11465-11475.

63 K. T. Kim, J. Park, S. J. Jo, S. Jung, O. S. Kwon, G. P. Gallerano, W. Y. Park and G. S. Park, Sci. Rep., 2013, 3, 2296.

64 V. F. Kirichuck, A. N. Ivanov, O. N. Antipova, A. P. Krenickiy, A. V. Mayborodin and V. D. Tupikin, Bull. Exp. Biol. Med., 2008, 145, 75-77.

65 N. Y. Weisman, V. I. Fedorov and E. F. Nemova, Contemporary Problems of Ecology, 2015, 8, 237-242.

66 D. R. Dalzell, J. Mcquade, R. Vincelette, B. Ibey, J. Payne, R. Thomas, W. P. Roach, C. L. Roth and G. J. Wilmink, Proc. SPIE-Int. Soc. Opt. Eng., 2010, 7562, 75620.

67 W. Rachel, S. Amy, H. Gareth, D. Joan, E. David, H. Paul, S. K. Michele, S. Mark, D. David and H. Stephen, Phys. Med. Biol., 2013, 58, 373-391.

68 J. W. Gerald, D. R. Benjamin, C. R. Caleb, L. I. Bennett, A. P. Jason, X. C. Luisiana, E. G. Jessica, P. Xomalin, G. M. Dustin and P. R. William, Lasers Surg. Med., 2015, 43, 152-163.

69 A. R. Orlando and G. P. Gallerano, Int. J. Infrared Millimeter Waves, 2009, 30, 1308-1318. 
70 L. V. Titova, A. K. Ayesheshim, A. Golubov, D. Fogen, R. Rodriguez-Juarez, F. A. Hegmann and O. Kovalchuk, Biomed. Opt. Express, 2013, 4, 559-568.

71 B. S. Alexandrov, V. Gelev, A. R. Bishop, A. Usheva and K. Ø. Rasmussen, Phys. Lett. A, 2009, 374, 1214-1217.

72 B. S. Alexandrov, M. L. Phipps, L. B. Alexandrov, L. G. Booshehri, A. Erat, J. Zabolotny, C. H. Mielke, H. T. Chen, G. Rodriguez and K. Ø. Rasmussen, Sci. Rep., 2013, 3, 1184.
73 O. P. Cherkasova, V. I. Fedorov, E. F. Nemova and A. S. Pogodin, Opt. Spectrosc., 2009, 107, 534-537.

74 A. N. Bogomazova, E. M. Vassina, T. N. Goryachkovskaya, V. M. Popik, A. S. Sokolov, N. A. Kolchanov, M. A. Lagarkova, S. L. Kiselev and S. E. Peltek, Sci. Rep., 2015, 5, 7749.

75 L. Yu, M. Tang, L. Xia, W. Yu and W. Fu, RSC Adv., 2017, 7, 53963-53969. 